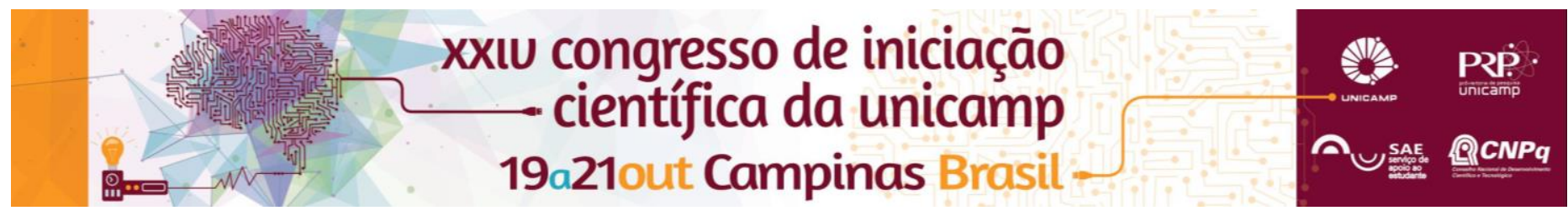

\title{
Biotransformation potential of phenylpropanoids synthesized by citrus host when infected by pathogens.
}

\author{
Maria L. Vieira*, Taícia P. Fill
}

\section{Resumo}

The culture of Geotrichum candidum along with phenylpronoids as cinnamic acid (which are substrates produced by the host as a defense mechanism against the fungus) led to different biotransformation products. Benzoic acid, one of the biotransformation products showed less toxicity against the fungus compared to the initial substrate.

\section{Palavras-chave:}

Biotransformation, Natural products, phenylpropanoids

\section{Introduction}

Brazil is the citrus largest producer and orange juice exporter in the world, therefore citriculture has a great impact on Brazilian economy. Citrus fruits are very susceptible to infections due to microbial pathogenic agents, leading to important economic losses during the postharvest phase ${ }^{1}$.

The most common and serious diseases that affect citrus are green and blue moulds caused, respectively, by Penicillium digitatum and Penicillium italicum and the sour rot, caused by the fungus Geotrichum candidum.

The research concerning such fungi focuses on treatments against the infection symptoms, nevertheless, literature about its secondary metabolites and biotransformation potential is scarce.

Citrus fruits when infected are able to produce compounds on its surface that are toxic to the fungus and are used as a plant defense mechanism. On the other hand, the fungus has a complex enzymatic system able to perform biotransformation of such compounds in order to make them less harmful, generating new less toxic biotransformation products.

In this sense, this research aims to make some progress on the knowledge concerning Geotrichum candidum biotransformation potential and its ability to perform chemical modifications in phenylpropanoids (cinnamic acid) produced by citrus as a defense mechanism.

\section{Results and Discussion}

In order to evaluate the biotransformation potential of the defense metabolites produced by the host citrus, the fungus $G$. candidum was cultured in Czapeck media supplemented with cinnamic acid during one week.

The cultures were filtered and the biotransformation products extracted with ethyl acetate, and submitted to HPLC/MS analysis.

Based on the data obtained it was possible to verify the fungus ability to perform different biotransformation reactions indicating the great enzymatic machinery of Geotrichum candidum. Based on these results a scale-up experiment was performed in order to purify and further characterize the biotransformation products. The extract obtained was submitted to a classical column chromatography, with a linear gradient of methanol and ethyl acetate.

The major biotransformation products were determined to be benzoic acid and p-coumaric acid which were characterized by spectroscopic techniques (Figure 1).
Other biotransformation products detected are under investigation in our laboratory.

To verify the toxicity of the biotransformation product which could indicate a fungus adaptation mechanism against cinnamic acid produced in vivo by citrus, the fungus was cultivated in the presence of cinnamic acid; benzoic acid and with no additional compounds (control), in PDA solid media.

The results to this experiment can be observed in the figure $1 \mathrm{~B}$ below.

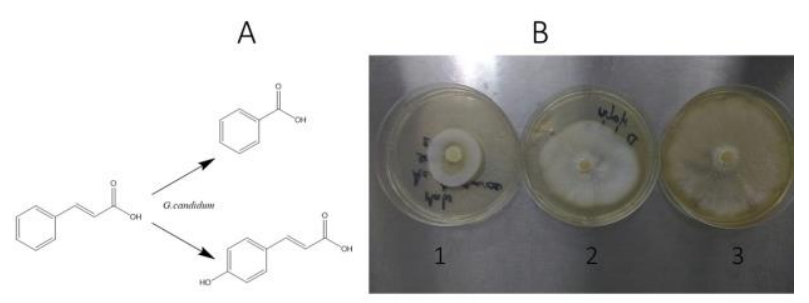

Figure 1. A) Biotransformation products obtained. B) 1- G. candidum in PDA with $300 \mu \mathrm{L}$ of cinnamic acid $(250 \mathrm{mg} / \mu \mathrm{L}) ; 2$ G.candidum in PDA with $300 \mu \mathrm{L}$ of the product $(250 \mathrm{mg} / \mu \mathrm{L}) ; 33$ Control.

As observed in the figure $1 \mathrm{~B}$, the culture 1 with cinnamic acid had the lowest growth, when compared to the control experiment. This proves that cinnamic acid has significant toxicity to the fungus studied. On the other hand, the culture with benzoic acid showed considerable less toxicity compared to cinnamic acid, suggesting that the fungus was able to perform a chemical modification in order to adapt to the new medium conditions. In vivo experiments will be performed to evaluate the fungus ability to perform such reactions in the fruit and understand $G$. candidum ability to infect citrus fruits.

\section{Conclusions}

Considering that the post-harvest diseases are responsible for most losses in the production of citrus, it is of great importance to develop new studies on this subject. Through the experiments with cinnamic acid it has been possible to study the biotransformation potential of the fungus and gain insights about the fungus ability to infect the host .

${ }^{1}$ Snowden, A. L. Post-Harvest diseases and disorders and vegetables: Volume 1: General Introduction and fruits. Iowa: CRC Press, 2008

${ }^{2}$ A.-R. Ballester et al. Citrus phenylpropanoids and defence against pathogens. Part II: Gene expression and metabolite accumulation in the response of fruits to Penicillium digitatum infection. Food Chemistry; 136 (2013), p. 285-291 ${ }^{3}$ Hegazy M. E., Mohamed T. A., ElShamy A. I., Mohamed A. E., Mahalel U. A., Reda E. H., et al. Microbial biotransformation as a tool for drug development based on natural products from mevalonic acid pathway: a review. Journal of Advanced Research; 6 (2015), 17-33. 